\title{
The metabolic signature associated with the Western dietary pattern: a cross-sectional study
}

\author{
Annie Bouchard-Mercier ${ }^{1,2,3}$, Iwona Rudkowska ${ }^{1,3}$, Simone Lemieux ${ }^{1,2}$, Patrick Couture ${ }^{1,3}$ and Marie-Claude Vohl $1^{1,2,3^{*}}$
}

\begin{abstract}
Background: Metabolic profiles have been shown to be associated to obesity status and insulin sensitivity. Dietary intakes influence metabolic pathways and therefore, different dietary patterns may relate to modifications in metabolic signatures. The objective was to verify associations between dietary patterns and metabolic profiles composed of amino acids (AAs) and acylcarnitines (ACs).

Methods: 210 participants were recruited in the greater Quebec City area between September 2009 and December 2011. Dietary patterns had been previously derived using principal component analysis (PCA). The Prudent dietary pattern was characterised by higher intakes of vegetables, fruits, whole grain products, non-hydrogenated fat and lower intakes of refined grain products, whereas the Western dietary pattern was associated with higher intakes of refined grain products, desserts, sweets and processed meats. Targeted metabolites were quantified in 37 participants with the Biocrates Absolute IDQ p150 (Biocrates Life Sciences AG, Austria) mass spectrometry method (including 14 amino acids and 41 acylcarnitines).

Results: PCA analysis with metabolites including AAs and ACs revealed two main components explaining the most variance in overall data (13.8\%). PC1 was composed mostly of medium- to long-chain ACs (C16:2, C14:2, C14:2-OH, C16, C14:1-OH, C14:1, C10:2, C5-DC/C6-OH, C12, C18:2, C10, C4:1-DC/C6, C8:1 and C2) whereas PC2 included certain AAs and short-chain ACs (xLeu, Met, Arg, Phe, Pro, Orn, His, C0, C3, C4 and C5). The Western dietary pattern correlated negatively with PC1 and positively with PC2 $(r=-0.34, p=0.05$ and $r=0.38, p=0.03$, respectively), independently of age, sex and BMI.
\end{abstract}

Conclusion: These findings suggest that the Western dietary pattern is associated with a specific metabolite signature characterized by increased levels of AAs including branched-chain AAs (BCAAs) and short-chain ACs.

Trial registration: NCT01343342

Keywords: Dietary pattern, Western dietary pattern, Prudent dietary pattern, Acylcarnitine, Amino acids, Branched-chain amino acids, Metabolites

\section{Background}

Single nutrients or single food components have been frequently studied in order to achieve a better understanding of their impact on health and on the development of chronic diseases. Many studies have also observed the effects of global diets or dietary patterns such as the Mediterranean diet on chronic diseases. Studying the effects of dietary patterns takes into account the interactions

\footnotetext{
* Correspondence: marie-claude.vohl@fsaa.ulaval.ca

${ }^{1}$ Institute of Nutrition and Functional Foods (INAF), Laval University, 2440

Hochelaga Blvd., Quebec G1V 0A6, Canada

${ }^{2}$ Department of Food Science and Nutrition, Laval University, 2425 de

I'Agriculture St, Quebec G1K 7P4, Canada

Full list of author information is available at the end of the article
}

between nutrients [1]. Dietary patterns derived by principal component analysis (PCA) depict a portrait of the foods that are mainly consumed together within a population [1]. Dietary patterns, such as the Prudent (or Healthy pattern) and the Western dietary patterns have been associated positively or inversely with cardiovascular disease risk factors and mortality, as well as with certain types of cancer such as colorectal cancer [2-4]. Dietary patterns have also been associated with type 2 diabetes or related metabolic parameters. Schulze et al. [5] observed an association between the risk of type 2 diabetes and a dietary pattern high in foods such as sugar-sweetened soft drinks, refined grains as well as processed meats and 
low vegetable intake. In addition, Heidemann et al. [6] reported a decreased risk of type 2 diabetes with a dietary pattern characterised by high intakes of fruits and low intakes of foods such as high-caloric soft drinks and meats. These results have been confirmed by Esposito et al. [7] in a recent systematic review which observed that dietary patterns characterized by high intakes of fruit and vegetables, whole grains, fish, and poultry, and low intakes of red meat, processed foods, sugar-sweetened beverages, and starchy foods were associated with a reduced risk and a later development of type 2 diabetes.

Obesity pandemic represents a major health burden. In Canada, $26 \%$ of the adults were considered obese according to their body mass index (BMI) and when considering waist circumference, $37 \%$ were abdominally obese [8]. Obesity is closely related to insulin resistance [9]. However, the link between these two conditions is not well understood. Studying metabolites may help in further understanding the effects of diets, drugs and diseases at the cellular level and enhance our comprehension of the development of complex diseases such as type 2 diabetes (or insulin resistance) [10]. A few studies have investigated metabolic signatures in relation to insulin sensitivity or obesity [11-14]. For example, Newgard et al. [12] have observed a metabolic signature including branched-chain amino acids (BCAA) and short-chain acylcarnitines (ACs) among obese and insulin resistant individuals. These observations have also been replicated in Chinese and Asian-Indian individuals where a metabolic signature characterised by higher concentrations of amino acids (AAs) such as leucine/isoleucine, phenylalanine, tyrosine and methionine was also associated with insulin resistance [13]. Other studies observed the effects of dietary variables on metabolic signatures including ACs. For example, the effects of a lactovegetarien diet versus an omnivorous diet, the intake of fruits and vegetables, coffee and garlic intakes and hypocaloric dieting were studied [15-17]. Dietary patterns derived from cluster analysis have also been associated with specific metabolites [18].

To our knowledge, the metabolic signatures associated with the Western and the Prudent dietary patterns have never been studied. Therefore, the objective of this study was to investigate the metabolic signatures, composed of AAs and ACs derived from PCA, associated with the Western and the Prudent dietary patterns in a sample of overweight men and women. Associations between the Western dietary pattern and principal components (PCs) were observed.

\section{Methods}

\section{Subjects}

A total of 254 subjects were recruited to participate in this clinical trial from the greater Quebec City metropolitan area between September 2009 and December 2011 through advertisements in local newspapers as well as by electronic messages sent to university students/employees. To be eligible, subjects had to be non-smokers and free of any thyroid or metabolic disorders requiring treatment such as diabetes, hypertension, severe dyslipidemia, and coronary heart disease. Participants had to be aged between 18 and 50 years with a BMI between 25 and $40 \mathrm{Kg} / \mathrm{m}^{2}$. The subjects who had taken $\mathrm{n}-3$ polyunsaturated fatty acid supplements during the six months preceding the study were excluded. A total of 210 subjects completed the study protocol which is described elsewhere [19] and were included in this cross-sectional study. All participants gave written informed consent and the experimental protocol was approved by the ethics committees of Laval University Hospital Research Center and Laval University. This trial was registered at clinicaltrials.gov as NCT01343342.

\section{Dietary assessment and food grouping}

Dietary assessment and food grouping has been previously described [20]. Briefly, dietary intake of the past month was determined by a 91-items validated food frequency questionnaire (FFQ) [21] based on food habits of Quebecers, administered by a registered dietitian (RD). All the information was compiled and similar food items from the FFQ were grouped, as previously described [22]. Three criteria were used to form these groups: first, the similarity of nutrient profiles, second, the culinary usage of different types of food (similar to groups used in a previous study [23]) and third, the consideration of groups utilized in other studies to maintain consistency [1]. Food items from only thirty-five food groups were consumed by the participants in the present study. From these thirtyfive food groups, eight were not normally distributed even after logarithmic transformation and were excluded as well. Consequently, twenty-seven foods groups were used for PCA to generate dietary patterns as described previously [20]. Briefly, two main dietary patterns were derived from PCA analysis. The Prudent dietary pattern, characterized by higher intakes of vegetables, fruits, whole grain products, non-hydrogenated fat and lower intakes of refined grain products, and the Western dietary pattern, associated with higher intakes of refined grain products, desserts, sweets and processed meats [20].

\section{Metabolite profiling}

The Biocrates Absolute IDQ p150 (Biocrates Life Sciences AG, Austria) mass spectrometry method was used to quantify 163 metabolites for the first 40 of the 254 participants. Three participants were excluded because of extreme values (standard out of range), resulting in 37 participants. For this study ACs and AAs were the main focus thus, $41 \mathrm{ACs}$ (ACx:y, where $x$ denotes the number 
of carbons in the side chain and $y$ the number of double bonds) and 14 AAs (proteinogenic + ornithine) were studied. Assays used $10 \mu \mathrm{L}$ of plasma from each subject. The metabolite profiling was carried out according to the manufacturer's instructions at CHENOMX (Edmonton, AL, Canada). For all analyzed metabolites the concentrations are reported in $\mu \mathrm{M}$. Furthermore, metabolites with standard out of range and/or for which more than half of the values were below the limit of detection were excluded. Thus, 29 ACs and 13 AAs were included in the analyses.

\section{Statistical analyses}

Variables which were not normally distributed were logarithmically transformed. The distribution of glutaconyl-Lcarnitine (C5_1_DC) was still not normally distributed after logarithmic transformation and thus was excluded from further analyses. The FACTOR procedure from Statistical Analysis Software (SAS) using PCA method was used to derive PCs describing metabolite signatures. Newgard et al. [11] described two main PCs when studying ACs and AAs which explained most of the variance in their data. In the present study, in order to determine the number of factors to retain, components with eigenvalue $>1$, values at Scree test, variance explained (\%) and the interpretability were considered. It was noticed that PC1 and PC2 had eigenvalues much higher $(\sim 8$ and $\sim 6$, respectively) than the other PCs $(<\sim 3)$. Thus, the NFACTORS statement was added in the proc FACTOR procedure in order to retain only 2 main PCs and explain a maximum of variance. Metabolites with absolute factor loadings $\geq 0.50$ were regarded as significant contributors to the PC. Using the SCORE procedure of SAS, each participant was given a score for each PC. These scores are calculated from the sum of metabolic signature groups multiplied by their respective factor loading. These scores reflect the degree of each participant's metabolic signature conforming to PC1 and PC2.

Pearson correlations were used to detect associations between the Prudent and the Western dietary pattern scores with PC1 and PC2 scores. To further understand the relationships with $\mathrm{PC} 1$ and $\mathrm{PC} 2$ scores and dietary variables, partial correlations were performed with individual food groups (only the food groups which contributed to Prudent and Western dietary patterns) and macronutrients (expressed as energy percentages) adjusted for age, sex, BMI and energy intakes (only for the food groups). To facilitate interpretation, Prudent and Western dietary pattern scores as well as with food groups and macronutrients intakes were divided according to tertiles and associations with PC1 and PC2 were tested using the General Linear Model procedure implemented in SAS. A p-value $<0.05$ was considered significant. All statistical analyses were performed using SAS statistical software version 9.3 (SAS Institute, Inc., Cary, NC, USA).

\section{Results}

Descriptive characteristics and dietary patterns

Descriptive characteristics of the study participants are presented in Table 1. As described previously, there were two dietary patterns derived in this cohort, the Prudent and the Western dietary patterns [20]. The Prudent dietary pattern was characterised by high intakes of vegetables, fruits, whole grain products, non-hydrogenated fats and inversely associated with refined grain products food group and the Western dietary pattern by high intakes of refined grain products, desserts, sweets and processed meats [20]. In Table 2, dietary intakes, AC and AA concentrations according to dietary pattern score (low $\leq 0$ or high $>0$ ) are shown respectively for the Prudent and the Western dietary patterns. Individuals with high scores for the Prudent dietary pattern had lower saturated fat intakes than individuals with low scores. The opposite was observed for the Western dietary pattern scores. Regarding the associations between dietary patterns and cardiovascular disease risk factors, only a trend was observed for lower fasting insulin levels with higher scores for the Prudent dietary pattern $(\mathrm{r}=-0.32, \mathrm{p}=0.07)$. A positive association between fasting glucose levels and the Western dietary pattern was observed $(r=0.38$, $\mathrm{p}=0.03)$.

\section{Principal component analysis of the metabolites}

PC1 explained $7.97 \%$ of the variance in the data and $\mathrm{PC} 2$, 5.81\%. As presented in Figure 1, PC1 was composed mostly of medium- to long-chain ACs (C16:2, C14:2, C14:2-OH, C16, C14:1-OH, C14:1, C10:2, C5-DC/C6-OH,

Table 1 Descriptive characteristics of the study participants

\begin{tabular}{lc}
\hline Variables & All participants $(\mathbf{n}=\mathbf{3 7})$ \\
\hline Age (years) & $34.59 \pm 9.16$ \\
Sex (men/women) & $16 / 21$ \\
BMI $\left(\mathbf{K g} / \mathbf{m}^{\mathbf{2}}\right.$ ) & $29.69 \pm 4.17$ \\
Waist circumference (cm) & $93.46 \pm 11.89$ \\
Systolic blood pressure $(\mathbf{m m H g})$ & $108.03 \pm 8.67$ \\
Diastolic blood pressure $(\mathbf{m m H g})$ & $71.89 \pm 8.57$ \\
Fasting glucose (mmol/L) & $5.01 \pm 0.74$ \\
Fasting insulin (pmol/L) & $84.57 \pm 34.78$ \\
Total-C (mmol/L) & $5.29 \pm 1.35$ \\
LDL-C (mmol/L) & $3.18 \pm 1.13$ \\
HDL-C (mmol/L) & $1.46 \pm 0.48$ \\
Triglycerides (mmol/L) & $1.43 \pm 0.93$ \\
ApoB (g/L) & $0.96 \pm 0.30$ \\
\hline
\end{tabular}

Means \pm SD. 
Table 2 Dietary intakes and plasma AC and AA according to dietary pattern score

\begin{tabular}{|c|c|c|c|c|c|c|}
\hline & $\begin{array}{l}\text { Low Prudent }(\leq 0) \\
(n=20)\end{array}$ & $\begin{array}{l}\text { High Prudent }(>0) \\
(n=17)\end{array}$ & P-value ${ }^{1}$ & $\begin{array}{c}\text { Low Western }(\leq 0) \\
(n=16)\end{array}$ & $\begin{array}{l}\text { High Western }(>0) \\
(n=21)\end{array}$ & P-value ${ }^{1}$ \\
\hline \multicolumn{7}{|l|}{ Dietary intakes } \\
\hline Carbohydrate (\%) & $49.38 \pm 5.99$ & $50.48 \pm 6.37$ & 0.35 & $51.91 \pm 7.21$ & $48.35 \pm 4.73$ & 0.35 \\
\hline Protein (\%) & $17.14 \pm 2.78$ & $18.46 \pm 2.26$ & 0.20 & $18.20 \pm 2.66$ & $17.40 \pm 2.58$ & 0.43 \\
\hline Total fat (\%) & $32.57 \pm 6.15$ & $31.93 \pm 4.19$ & 0.56 & $30.52 \pm 5.89$ & $33.61 \pm 4.45$ & 0.29 \\
\hline Saturated fat (\%) & $11.41 \pm 1.83$ & $9.55 \pm 2.11$ & 0.004 & $9.11 \pm 2.08$ & $11.65 \pm 1.47$ & 0.0006 \\
\hline Monounsaturated fat (\%) & $13.24 \pm 3.06$ & $13.48 \pm 2.04$ & 0.92 & $12.83 \pm 2.98$ & $13.75 \pm 2.28$ & 0.81 \\
\hline Polyunsaturated fat (\%) & $5.30 \pm 1.53$ & $6.24 \pm 1.30$ & 0.07 & $5.97 \pm 1.57$ & $5.55 \pm 1.44$ & 0.17 \\
\hline Cholesterol (mg) & $329.88 \pm 167.61$ & $357.86 \pm 188.90$ & 0.84 & $263.76 \pm 167.15$ & $402.92 \pm 160.78$ & 0.07 \\
\hline Total fiber (g) & $19.38 \pm 4.83$ & $30.43 \pm 7.03$ & $<0.0001$ & $25.71 \pm 7.53$ & $23.51 \pm 8.54$ & 0.31 \\
\hline \multicolumn{7}{|l|}{ Acylcarnitines (ACs) } \\
\hline CO & $25.16 \pm 5.07$ & $27.50 \pm 7.09$ & 0.24 & $23.78 \pm 6.73$ & $28.10 \pm 4.97$ & 0.24 \\
\hline C2 & $5.55 \pm 1.36$ & $5.20 \pm 1.55$ & 0.57 & $5.33 \pm 1.48$ & $5.44 \pm 1.44$ & 0.76 \\
\hline C3 & $0.36 \pm 0.14$ & $0.29 \pm 0.10$ & 0.04 & $0.30 \pm 0.12$ & $0.34 \pm 0.13$ & 0.95 \\
\hline C4 & $0.18 \pm 0.06$ & $0.16 \pm 0.07$ & 0.44 & $0.16 \pm 0.08$ & $0.18 \pm 0.05$ & 0.46 \\
\hline C4:1-DC/C6 & $0.05 \pm 0.01$ & $0.06 \pm 0.02$ & 0.08 & $0.05 \pm 0.02$ & $0.05 \pm 0.02$ & 0.39 \\
\hline C5 & $0.11 \pm 0.04$ & $0.11 \pm 0.03$ & 0.64 & $0.10 \pm 0.03$ & $0.12 \pm 0.04$ & 0.39 \\
\hline C5-DC/C6-OH & $0.01 \pm 0.00$ & $0.02 \pm 0.00$ & 0.01 & $0.01 \pm 0.01$ & $0.01 \pm 0.00$ & 0.62 \\
\hline C8:1 & $0.14 \pm 0.08$ & $0.12 \pm 0.06$ & 0.63 & $0.12 \pm 0.08$ & $0.13 \pm 0.07$ & 0.83 \\
\hline C10 & $0.16 \pm 0.07$ & $0.22 \pm 0.13$ & 0.07 & $0.17 \pm 0.09$ & $0.20 \pm 0.11$ & 0.47 \\
\hline C10:2 & $0.02 \pm 0.01$ & $0.03 \pm 0.01$ & 0.11 & $0.02 \pm 0.01$ & $0.03 \pm 0.01$ & 0.12 \\
\hline C12 & $0.06 \pm 0.03$ & $0.08 \pm 0.03$ & 0.06 & $0.06 \pm 0.03$ & $0.08 \pm 0.03$ & 0.31 \\
\hline C14:1 & $0.17 \pm 0.03$ & $0.21 \pm 0.09$ & $<0.05$ & $0.19 \pm 0.08$ & $0.19 \pm 0.07$ & 0.95 \\
\hline $\mathrm{C} 14: 1-\mathrm{OH}$ & $0.01 \pm 0.00$ & $0.01 \pm 0.01$ & 0.54 & $0.01 \pm 0.01$ & $0.01 \pm 0.01$ & 0.41 \\
\hline C14:2 & $0.03 \pm 0.01$ & $0.04 \pm 0.02$ & 0.02 & $0.03 \pm 0.02$ & $0.03 \pm 0.02$ & 0.89 \\
\hline $\mathrm{C} 14: 2-\mathrm{OH}$ & $0.01 \pm 0.00$ & $0.01 \pm 0.01$ & 0.19 & $0.01 \pm 0.00$ & $0.01 \pm 0.00$ & 0.63 \\
\hline C16 & $0.08 \pm 0.02$ & $0.08 \pm 0.04$ & 0.37 & $0.07 \pm 0.03$ & $0.08 \pm 0.03$ & 0.45 \\
\hline C16:2 & $0.01 \pm 0.00$ & $0.01 \pm 0.01$ & 0.19 & $0.01 \pm 0.00$ & $0.01 \pm 0.01$ & 0.65 \\
\hline C18:2 & $0.04 \pm 0.01$ & $0.05 \pm 0.01$ & 0.002 & $0.04 \pm 0.01$ & $0.04 \pm 0.01$ & 0.42 \\
\hline \multicolumn{7}{|l|}{ Amino acids } \\
\hline xleucine & $183.95 \pm 46.81$ & $161.29 \pm 26.70$ & 0.03 & $152.94 \pm 21.48$ & $189.24 \pm 44.08$ & 0.03 \\
\hline Methionine & $28.37 \pm 5.14$ & $26.54 \pm 4.32$ & 0.05 & $24.25 \pm 4.23$ & $30.02 \pm 3.60$ & 0.0004 \\
\hline Arginine & $99.91 \pm 32.87$ & $95.81 \pm 23.14$ & 0.64 & $93.54 \pm 27.05$ & $101.44 \pm 29.76$ & 0.79 \\
\hline Phenylalanine & $48.18 \pm 8.10$ & $46.56 \pm 4.91$ & 0.31 & $43.86 \pm 4.55$ & $50.16 \pm 7.02$ & 0.007 \\
\hline Proline & $167.32 \pm 58.74$ & $157.68 \pm 39.76$ & 0.47 & $154.47 \pm 44.03$ & $169.30 \pm 55.06$ & 0.89 \\
\hline Ornitine & $49.06 \pm 17.18$ & $52.61 \pm 15.80$ & 0.48 & $45.44 \pm 17.75$ & $54.69 \pm 14.51$ & 0.33 \\
\hline Histidine & $93.59 \pm 20.35$ & $93.79 \pm 13.96$ & 0.94 & $93.55 \pm 16.26$ & $93.78 \pm 17.74$ & 0.65 \\
\hline
\end{tabular}

Means \pm SD.

${ }^{1} \mathrm{P}$-values of the GLM models are adjusted for age, sex and BMI.

$\mathrm{P}$-values in bold were considered significantly different.

C12, C18:2, C10, C4:1-DC/C6, C8:1 and C2) whereas PC2 was mainly composed of AAs and short-chain ACs (xLeu, Met, Arg, Phe, Pro, Orn, His, C0, C3, C4 and C5).

The Prudent dietary pattern was not correlated neither to PC1 nor to PC2 $(r=-0.19, p=0.26$ and $r=-0.21$, $\mathrm{p}=0.21$, respectively). The Western dietary pattern, was correlated with $\mathrm{PC} 2(\mathrm{r}=0.34, \mathrm{p}=0.04)$. When further adjusted for the effects of age, sex and BMI, both PCs were associated with the Western dietary pattern. The Western dietary pattern correlated negatively with $\mathrm{PC} 1$ and positively with $\mathrm{PC} 2(\mathrm{r}=-0.34, \mathrm{p}=0.05$ and $\mathrm{r}=0.38, \mathrm{p}=0.03$, respectively). To further explore the association between 


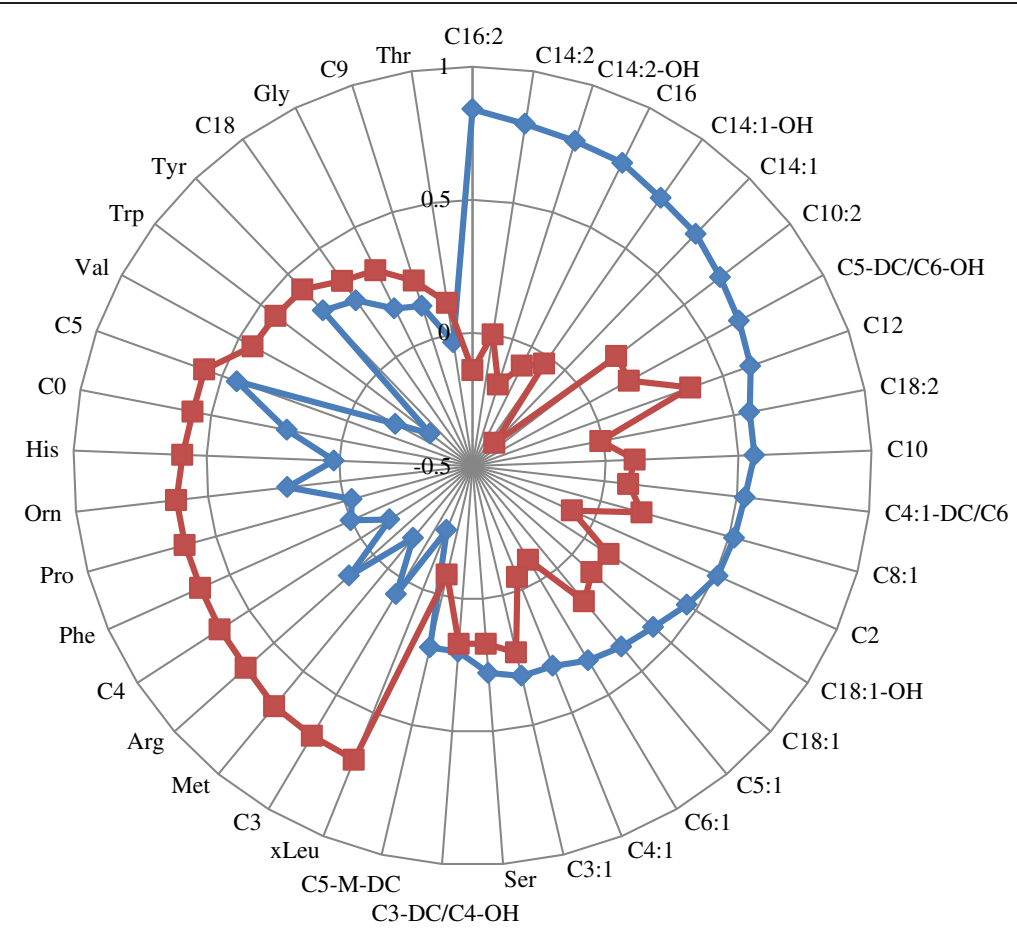

Figure 1 ACs and AAs associated with PC1 and PC2. Metabolites with absolute factor loadings $\geq 0.50$ were regarded as significant contributors to the PC. The blue line and squares represent PC1 and the red line and squares represent PC2.

the Western dietary pattern and PCs, subjects were divided into tertiles according to the Western dietary pattern score. The relationships between tertiles of the Western dietary pattern and PC1 and PC2 scores were not significant $(\mathrm{p}=0.10$ and $\mathrm{p}=0.15$, respectively).

In order to achieve a better understanding of the impact of dietary variables on the metabolic signature, the possible correlations between each dietary variable (food groups contributing either to Prudent or Western dietary pattern and macronutrient intake (expressed in energy percentages)) and the scores for each PC were tested (adjusted for age, sex, BMI and energy intakes (only for food groups)), as presented in Table 3. Briefly, PC1 was not correlated with any food groups. PC2 was negatively correlated with fruit intake and positively associated with dessert intake $(r=-0.38, p=0.03$ and $r=0.37$, $\mathrm{p}=0.04$, respectively) adjusted for age, sex, BMI and energy intakes. For the macronutrients, expressed as energy percentages, a positive association for PC2 with total fat and saturated fat intakes was observed $(r=0.39, p=0.02$ and $\mathrm{r}=0.50, \mathrm{p}=0.003$, respectively). Interestingly, when dietary total fat, saturated fat, fruit and dessert were divided into tertiles, only saturated fat intake tertiles were different according to PC2 scores (Figure 2) $(\mathrm{p}=0.01)$. As shown in Figure 2, saturated fat intakes $\leq 11.30 \%$ had negative PC2 scores, which indicate that their metabolic signature was not corresponding to $\mathrm{PC} 2$ characterised by higher concentrations of ACs and short-chain AAs. PC1 did not correlate with any macronutrients. In Table 4, partial correlations between dietary pattern scores and each $\mathrm{AC}$ and AA (only the metabolites which were associated with a PC) are shown. The Prudent dietary pattern score was positively associated with concentrations of C5-DC/ C6-OH (glutaryl-L-carnitine) and C18:2 (octadecadienylL-carnitine). The Western dietary pattern score was positively associated with methionine and phenylalanine.

\section{Dietary intakes and individual metabolites}

To further explore the impact of dietary intakes on metabolites, partial correlations were tested for each metabolite. Briefly, intakes of vegetables and fruits were positively associated with C18:2 (octadecadienyl-L-carnitine) $(r=0.49$, $\mathrm{p}=0.004$, for both) and inversely associated with xleucine $(r=-0.35, p=0.05$, for both). Fruit intakes were also inversely associated with methionine $(\mathrm{r}=-0.40, \mathrm{p}=0.02)$. The intakes of non-hydrogenated fats were positively associated with C14:1 (tetradecadienyl-L-carnitine) and C18:2 (octadecadienyl-L-carnitine) $(\mathrm{r}=0.39, \mathrm{p}=0.02$ and $\mathrm{r}=$ $0.46, p=0.007$, respectively) and inversely with histidine $(\mathrm{r}=-0.42, \mathrm{p}=0.01)$. The intakes of dessert were positively associated with three AAs, methionine, phenylalanine and xleucine $(\mathrm{r}=0.49, \mathrm{p}=0.004, \mathrm{r}=0.40, \mathrm{p}=0.02$ and $\mathrm{r}=0.38$, $\mathrm{p}=0.03$, respectively). The intake of sweets also was associated with methionine concentrations $(\mathrm{r}=0.41, \mathrm{p}=0.02)$ and with $\mathrm{C} 18: 1-\mathrm{OH}$ (hydroxyoctadecenoyl-L-carnitine) and C5:1-DC (glutaconyl-L-carnitine) $(r=0.37, p=0.04$ 
Table 3 Partial correlations between metabolite PCs and dietary pattern scores, food groups and macronutrient intakes

\begin{tabular}{|c|c|c|c|}
\hline Dietary variables & & PC1 & PC2 \\
\hline \multicolumn{4}{|l|}{ Dietary patterns } \\
\hline \multirow[t]{2}{*}{ Prudent dietary pattern } & r & -0.18 & -0.25 \\
\hline & $p^{1}$ & 0.31 & 0.15 \\
\hline \multirow[t]{2}{*}{ Western dietary pattern } & r & -0.34 & 0.38 \\
\hline & $p^{1}$ & 0.05 & 0.03 \\
\hline \multicolumn{4}{|l|}{ Food groups } \\
\hline \multirow[t]{2}{*}{ Processed meats } & r & -0.14 & -0.12 \\
\hline & $p^{2}$ & 0.45 & 0.52 \\
\hline \multirow[t]{2}{*}{ Vegetables } & r & -0.13 & -0.27 \\
\hline & $p^{2}$ & 0.46 & 0.13 \\
\hline \multirow[t]{2}{*}{ Fruits } & r & -0.10 & -0.38 \\
\hline & $p^{2}$ & 0.59 & 0.03 \\
\hline \multirow[t]{2}{*}{ Whole grain products } & r & -0.21 & -0.24 \\
\hline & $p^{2}$ & 0.23 & 0.18 \\
\hline \multirow[t]{2}{*}{ Non-hydrogenated fats } & r & -0.02 & -0.17 \\
\hline & $p^{2}$ & 0.93 & 0.34 \\
\hline \multirow[t]{2}{*}{ Refined grain products } & r & -0.14 & 0.04 \\
\hline & $p^{2}$ & 0.43 & 0.85 \\
\hline \multirow[t]{2}{*}{ Desserts } & r & -0.09 & 0.37 \\
\hline & $p^{2}$ & 0.60 & 0.04 \\
\hline \multirow[t]{2}{*}{ Sweets } & r & -0.18 & 0.25 \\
\hline & $p^{2}$ & 0.32 & 0.16 \\
\hline \multicolumn{4}{|l|}{ Macronutrient intakes } \\
\hline \multirow[t]{2}{*}{ Total fat (\%) } & r & 0.23 & 0.39 \\
\hline & $p^{1}$ & 0.20 & 0.02 \\
\hline \multirow[t]{2}{*}{ Saturated fat (\%) } & r & 0.11 & 0.50 \\
\hline & $p^{1}$ & 0.52 & 0.003 \\
\hline \multirow[t]{2}{*}{ Monounsaturated fat (\%) } & r & 0.28 & 0.28 \\
\hline & $p^{1}$ & 0.10 & 0.10 \\
\hline \multirow[t]{2}{*}{ Polyunsaturated fat (\%) } & r & 0.06 & 0.07 \\
\hline & $p^{1}$ & 0.74 & 0.70 \\
\hline \multirow[t]{2}{*}{ Protein (\%) } & r & -0.00 & -0.14 \\
\hline & $p^{1}$ & 0.99 & 0.42 \\
\hline \multirow[t]{2}{*}{ Carbohydrate (\%) } & r & -0.00 & -0.29 \\
\hline & $p^{1}$ & 0.99 & 0.10 \\
\hline
\end{tabular}

${ }^{1} \mathrm{P}$-values are adjusted for age, sex and BMI.

${ }^{2} \mathrm{P}$-values are adjusted for age, sex, BMI and energy intakes.

$\mathrm{P}$-values in bold were considered significantly different.

and $\mathrm{r}=0.42, \mathrm{p}=0.01$, respectively). When observing macronutrient intakes, saturated fat intakes expressed as energy percentages were positively associated with $\mathrm{C} 5$ (valeryl-L-carnitine) $(\mathrm{r}=0.36, \mathrm{p}=0.04)$ and inversely with C18:2 (octadecadienyl-L-carnitine) $(\mathrm{r}=-0.46, \mathrm{p}=0.006)$. Monounsaturated fat intakes were positively associated

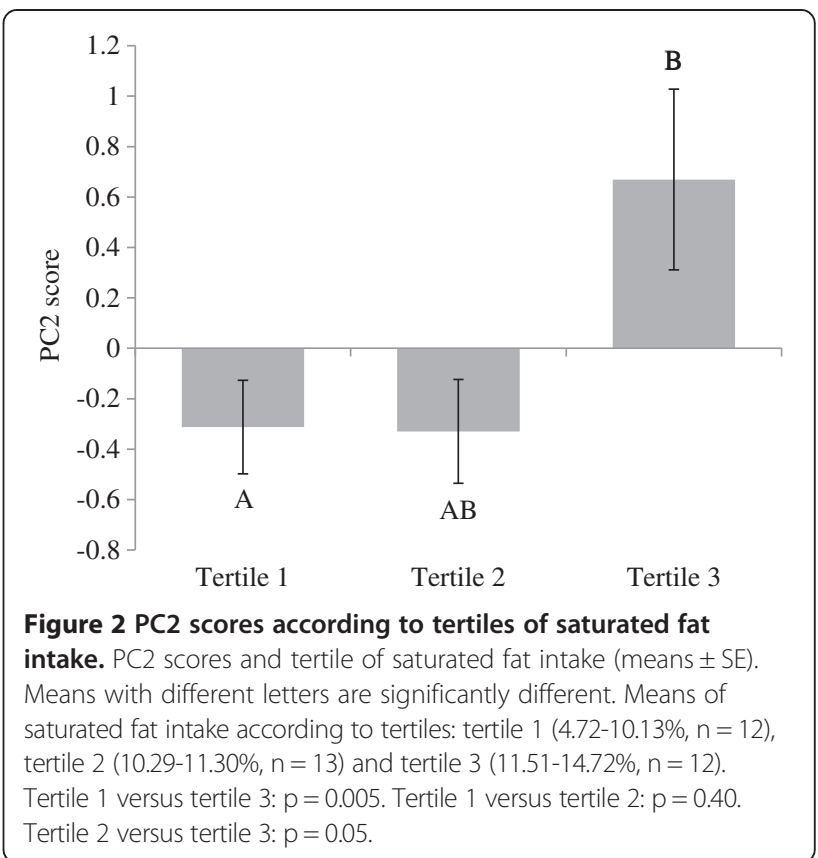

with C8:1 (octenoyl-L-carnitine) $(\mathrm{r}=0.50, \mathrm{p}=0.003)$ and inversely with C5-M-DC (methylglutaryl-L-carnitine) $(\mathrm{r}=-0.42, \mathrm{p}=0.01)$. Polyunsaturated fat intakes were also inversely associated with C5-M-DC (methylglutarylL-carnitine) $(\mathrm{r}=-0.35, \mathrm{p}=0.04)$ but as well with proline concentrations $(\mathrm{r}=-0.35, \mathrm{p}=0.04)$ and positively associated with C10:2 (decadienyl-L-carnitine) $(r=0.37$, $\mathrm{p}=0.03$ ). Protein intakes expressed as energy percentages were associated with ornithine and histidine $(r=0.44$, $\mathrm{p}=0.009$ and $\mathrm{r}=0.42, \mathrm{p}=0.01$, respectively). The opposite was observed for carbohydrate intakes which correlated inversely with ornithine concentrations $(r=-0.55$, $\mathrm{p}=0.0008)$.

\section{Discussion}

The Prudent and the Western dietary patterns from this study had many similarities with the dietary patterns described in the literature. The Prudent dietary pattern is usually associated with high consumption of vegetables, fruits and whole grain products whereas the Western dietary pattern relates to higher intakes of red and processed meats, refined grain products and sweets [24]. In this study, an association with higher fasting glucose was observed among individuals with high scores for the Western dietary pattern. Associations between dietary patterns and type 2 diabetes have been frequently observed and have been reviewed recently by Alhazmi et al. [25].

PC1 was composed mainly of medium- to long-chain ACs whereas PC2 was composed of short-chain ACs and AAs including the BCAA xleucine and the aromatic AA phenylalanine. Recent studies have shown a link 
Table 4 Partial correlations between dietary pattern scores, ACs and AAs

\begin{tabular}{|c|c|c|c|c|}
\hline \multirow{2}{*}{$\frac{\text { Acylcarnitines }}{\mathrm{CO}}$} & \multicolumn{2}{|c|}{ Prudent dietary pattern } & \multicolumn{2}{|c|}{ Western dietary pattern } \\
\hline & r & 0.09 & $r$ & 0.18 \\
\hline & $p$ & 0.60 & p & 0.32 \\
\hline \multirow[t]{2}{*}{$\mathrm{C} 2$} & r & -0.05 & r & 0.07 \\
\hline & $p$ & 0.76 & p & 0.68 \\
\hline \multirow[t]{2}{*}{ C3 } & r & -0.23 & r & -0.02 \\
\hline & $\mathrm{p}$ & 0.20 & $\mathrm{p}$ & 0.92 \\
\hline \multirow[t]{2}{*}{ C4 } & r & -0.15 & $r$ & 0.15 \\
\hline & $p$ & 0.41 & $p$ & 0.41 \\
\hline \multirow[t]{2}{*}{$C 4: 1-D C / C 6$} & r & 0.26 & r & 0.13 \\
\hline & $p$ & 0.14 & $\mathrm{p}$ & 0.46 \\
\hline \multirow[t]{2}{*}{ C5 } & r & -0.26 & r & -0.02 \\
\hline & $p$ & 0.14 & $p$ & 0.91 \\
\hline \multirow[t]{2}{*}{$\mathrm{C} 5-\mathrm{DC} / \mathrm{C} 6-\mathrm{OH}$} & r & 0.35 & r & -0.06 \\
\hline & $p$ & 0.04 & $p$ & 0.74 \\
\hline \multirow[t]{2}{*}{ C8:1 } & r & -0.20 & r & -0.10 \\
\hline & $p$ & 0.25 & $p$ & 0.56 \\
\hline \multirow[t]{2}{*}{ C10 } & r & 0.16 & $r$ & 0.22 \\
\hline & $p$ & 0.36 & $\mathrm{p}$ & 0.20 \\
\hline \multirow[t]{2}{*}{$\mathrm{C} 10: 2$} & r & 0.19 & $r$ & 0.30 \\
\hline & $p$ & 0.29 & $p$ & 0.08 \\
\hline \multirow[t]{2}{*}{ C12 } & r & 0.18 & $r$ & 0.32 \\
\hline & $p$ & 0.32 & $p$ & 0.07 \\
\hline \multirow[t]{2}{*}{ C14:1 } & r & 0.27 & $r$ & 0.06 \\
\hline & $p$ & 0.12 & $p$ & 0.73 \\
\hline \multirow[t]{2}{*}{$\mathrm{C} 14: 1-\mathrm{OH}$} & r & 0.07 & $r$ & 0.11 \\
\hline & $p$ & 0.69 & $p$ & 0.52 \\
\hline \multirow[t]{2}{*}{ C14:2 } & r & 0.28 & $r$ & 0.08 \\
\hline & $p$ & 0.11 & $p$ & 0.67 \\
\hline \multirow[t]{2}{*}{$\mathrm{C} 14: 2-\mathrm{OH}$} & $r$ & 0.20 & $r$ & 0.05 \\
\hline & $p$ & 0.27 & $p$ & 0.78 \\
\hline \multirow[t]{2}{*}{ C16 } & r & 0.18 & r & 0.13 \\
\hline & $p$ & 0.30 & $p$ & 0.47 \\
\hline \multirow[t]{2}{*}{ C16:2 } & r & 0.15 & $r$ & 0.09 \\
\hline & $p$ & 0.39 & $p$ & 0.62 \\
\hline \multirow[t]{2}{*}{ C18:2 } & r & 0.51 & $r$ & -0.17 \\
\hline & $p$ & 0.002 & p & 0.34 \\
\hline
\end{tabular}

Amino acids

xleucine

Methionine

Arginine

$\begin{array}{ccc}-0.33 & r & 0.30 \\ 0.06 & p & 0.08 \\ -0.30 & r & 0.55 \\ 0.08 & p & \mathbf{0 . 0 0 0 8} \\ -0.22 & r & 0.22 \\ 0.21 & p & 0.21\end{array}$

Table 4 Partial correlations between dietary pattern scores, ACs and AAs (Continued)

\begin{tabular}{lcccc}
\hline Phenylalanine & $r$ & -0.13 & $r$ & 0.39 \\
Proline & $p$ & 0.46 & $p$ & $\mathbf{0 . 0 2}$ \\
& $r$ & -0.26 & $r$ & 0.03 \\
Ornitine & $p$ & 0.14 & $p$ & 0.87 \\
& $r$ & 0.16 & $r$ & -0.02 \\
Histidine & $p$ & 0.37 & $p$ & 0.92 \\
& $r$ & -0.25 & $r$ & -0.17 \\
\hline
\end{tabular}

P-values are all adjusted for age, sex and BMI.

P-values in bold were considered significantly different.

between plasma levels of certain AAs and the risk of insulin resistance. Newgard et al. [12] have observed a metabolic signature among obese individuals characterised by a combination of BCAA, methionine, aromatic AAs and short-chain ACs (C3 and C5) which was related to insulin resistance. It has also been observed that increased levels of BCAA and aromatic AAs were associated with the risk of developing future type 2 diabetes [26]. Laferrère et al. [11] have studied the impact on ACs and AAs of weight-loss induced by gastric bypass surgery or by a hypocaloric diet. In their study, the first PC (mostly medium- to long-chain ACs) was associated with improved insulin sensitivity and the second PC (mostly AAs and short-chain ACs) was associated with an increase in insulin resistance. The gastric bypass surgery was associated with a decrease in short-chain ACs: C3, C4-DC and C5 ACs and AAs: alanine, leucine/isoleucine, phenylalanine and tyrosine. The ACs $\mathrm{C} 3$ and $\mathrm{C} 5$ have been demonstrated to be, at least partly, the products of AAs catabolism, especially BCAA (leucine/isoleucine and valine), possibly indicating an increase in enzymes related to BCAA catabolism [12]. In this study, no correlations were observed between PCs and fasting insulin or glucose levels. This could be due to the fact that the individuals from this cohort were healthy as well as only slightly overweight. Thus, their metabolic profile was not deteriorated leading to too subtle differences to be detected according to PCs scores.

Interestingly, scores for the Western dietary pattern were inversely associated with PC1 (medium- to longchain ACs) and positively with $\mathrm{PC} 2$ (short-chain ACs and AAs including the BCAA xleucine). Xu et al. [15] compared the metabolite profile between a lactovegetarian diet with an omnivorous diet. Among the most different metabolites, there was glycine which was higher among lactovegeterians and phenylalanine which was lower among lactovegeterians compared to omnivorous controls. The authors hypothesised that phenylalanine concentrations may have been higher among the omnivorous group due to the intakes of animal proteins 
which contain more phenylalanine than proteins from vegetal sources. In our study, higher AAs levels in PC2 was not associated to an increase in total protein, animal protein or vegetal protein intakes (data not shown). In addition, protein intakes correlated positively with the Prudent dietary pattern and negatively with the Western dietary pattern. Thus, changes may be due to a modification in rates of protein turnover or AA catabolism. Differences in the expression of the enzyme responsible for BCAA catabolism (branched-chain $\alpha$-ketoacid dehydrogenase (BCKD) complex) have been reported among obese rats compared to their lean counterparts [27]. BCKD's activity was reduced among obese rats and also among diet induced obese mice, which were fed a diet containing from $45 \%$ to $60 \%$ energy from fat [27]. May et al. [16] have studied the effects on urine metabolomic profiles of a diet devoid of fruits and vegetables compared to a diet high in fruits and vegetables. They observed for the group deprived with fruits and vegetables, higher concentrations of short- to medium-chain ACs and higher concentrations of AAs and tricarboxylic cycle intermediates. They also hypothesised that these alterations could be due to a shift from glucose utilisation to fatty acid betaoxidation. In our study, the Western dietary pattern was inversely associated with vegetable consumption (data not shown) and with a PC (PC2) characterised by higher concentrations of four short-chain ACs and seven AAs. In addition, an inverse association between fruit consumption and PC2 was observed in the present study. Thus, it seems that low fruit and vegetable intakes may be associated with a metabolic signature characterised by higher levels of shorter chain ACs and AAs.

When further examining the relationships between food groups and PCs, the most important correlation was observed between saturated fat intakes and PC2. Mechanisms behind these relations are unknown. Saturated fat intakes have been shown to be less potent activators than polyunsaturated fatty acids of an important transcription factor regulating fatty acid beta-oxidation, peroxisome proliferator-activated receptor alpha (PPARA) [28]. It has also been observed that oleate, compared to palmitate, the main saturated fat from the diet, increased mitochondrial fatty acid beta-oxidation [29]. However, other studies have reported the opposite. Stephenson et al. [30] have observed that among rats fed a «Western» diet (higher in fat, saturated fat and sucrose intakes) the activity of several mitochondrial enzymes involved in fatty acid beta-oxidation was increased. These discrepancies may be dependent of the overall effect of diet. Saturated fat alone may not have the same effect on fuel selection than when consumed in conjunction with higher intakes of sugary foods. In rat models, it has been observed that in long term, the rats fed a diet high in saturated fat and sucrose developed more severe symptoms of the metabolic syndrome than rats fed diets either high in saturated fat or high in sucrose alone [31].

Even though this cohort was generally healthy, differences in metabolic signatures have been observed and may be indicative of a higher or lower risk of future cardiometabolic diseases. A strength of this study is the analysis of dietary intakes grouped in dietary patterns from FFQ which represents real life intakes. Obviously, functional analyses are needed to understand underlying mechanisms behind these associations between dietary patterns and ACs and AAs concentrations in the plasma. One limitation of this study could come from the use of PCA. Results could be sample specific and strongly affected by subjective analytic decisions [1]. Nevertheless, to minimize subjectivity and allow data to be used in other studies, eigenvalue, Scree test and the literature were examined before selecting the number of PCs and for the dietary patterns, data from previous studies were also considered for food grouping.

\section{Conclusion}

In conclusion, the results of the present study indicate a relationship between the Western dietary pattern and saturated fat intakes with a metabolic signature characterised by higher levels of short-chain ACs and AAs including BCAA and an aromatic AA. Individuals eating according to the Western dietary pattern or with high saturated fat intakes may increase their long term risk of cardiometabolic diseases possibly via small metabolic alterations.

\section{Abbreviations}

AA: Amino acid; AC: Acylcarnitine; Arg: Arginine; BCAA: Branched-chain amino acid; BCKD: Branched-chain a-ketoacid dehydrogenase; BMI: Body mass index; FFQ: Food frequency questionnaire; His: Histidine; Met: Methionine; Orn: Ornitine; PC: Principal component; PCA: Principal component analysis; Phe: Phenylalanine; PPARA: Peroxisome proliferator-activated receptor; Pro: Proline; RD: Registered dietitian; SAS: Statistical analysis software; $x$ Leu: xleucine.

\section{Competing interests}

The authors declare that they have no competing interest.

\section{Authors' contributions}

$\mathrm{IR}, \mathrm{SL}, \mathrm{PC}$, and MCV designed research; ABM conducted research with the research professionals; IR, SL, PC and MCV provided essential reagents or provided essential materials; ABM analyzed data and performed statistical analysis; ABM wrote paper; ABM, IR, SL, PC and MCV had primary responsibility for final content; All authors read and approved the final manuscript.

\section{Acknowledgments}

This research would not have been possible without the excellent collaboration of the participants. We would like to thank Hubert Cormier, Véronique Garneau, Alain Houde, Catherine Ouellette, Catherine Raymond, Élisabeth Thifault and the nurses, Danielle Aubin and Steeve Larouche, for their participation in the recruitment of the participants, the study coordination and the data collection.

ABM is supported by a studentship from the Fonds de recherche en santé du Quebec (FRQS) and by a Canadian Institutes of Health Research (CIHR) Frederick Banting and Charles Best Canada Graduate Scholarships Doctoral Awards (201210GSD-304012-190387), and MCV is Tier 1 Canada Research Chair in Genomics Applied to Nutrition and Health. This work was supported by a grant from CIHR - MOP-110975. 


\section{Author details}

'Institute of Nutrition and Functional Foods (INAF), Laval University, 2440 Hochelaga Blvd., Quebec G1V 0A6, Canada. ${ }^{2}$ Department of Food Science and Nutrition, Laval University, 2425 de l'Agriculture St, Quebec G1K 7P4, Canada. ${ }^{3}$ Endocrinology and Nephrology, CHU de Québec Research Center, 2705 Laurier Blvd., Québec G1V 4G2, Canada.

Received: 16 September 2013 Accepted: 6 December 2013 Published: 11 December 2013

\section{References}

1. Newby PK, Tucker KL: Empirically derived eating patterns using factor or cluster analysis: a review. Nutr Rev 2004, 62:177-203.

2. Bhupathiraju SN, Tucker KL: Coronary heart disease prevention: nutrients, foods, and dietary patterns. Clin Chim Acta 2011, 412:1493-1514.

3. Yusof AS, Isa ZM, Shah SA: Dietary patterns and risk of colorectal cancer: a systematic review of cohort studies (2000-2011). Asian Pac J Cancer Prev 2012, 13:4713-4717.

4. Esmaillzadeh A, Kimiagar M, Mehrabi Y, Azadbakht L, Hu FB, Willett WC: Dietary patterns, insulin resistance, and prevalence of the metabolic syndrome in women. Am J Clin Nutr 2007, 85:910-918

5. Schulze MB, Hoffmann K, Manson JE, Willett WC, Meigs JB, Weikert C, et al: Dietary pattern, inflammation, and incidence of type 2 diabetes in women. Am J Clin Nutr 2005, 82:675-684.

6. Heidemann C, Hoffmann K, Spranger J, Klipstein-Grobusch K, Mohlig M, Pfeiffer AF, et al: A dietary pattern protective against type 2 diabetes in the European Prospective Investigation into Cancer and Nutrition (EPIC)-Potsdam Study cohort. Diabetologia 2005, 48:1126-1134.

7. Esposito K, Kastorini CM, Panagiotakos DB, Giugliano D: Prevention of type 2 diabetes by dietary patterns: a systematic review of prospective studies and meta-analysis. Metab Syndr Relat Disord 2010, 8:471-476.

8. Janssen I: The public health burden of obesity in Canada. Can J Diabetes 2013, 37:90-96.

9. Abbasi F, Brown BW Jr, Lamendola C, McLaughlin T, Reaven GM: Relationship between obesity, insulin resistance, and coronary heart disease risk. J Am Coll Cardiol 2002, 40:937-943.

10. Ceglarek U, Leichtle A, Brugel M, Kortz L, Brauer R, Bresler K, et al: Challenges and developments in tandem mass spectrometry based clinical metabolomics. Mol Cell Endocrinol 2009, 301:266-271.

11. Laferrere B, Reilly D, Arias S, Swerdlow N, Gorroochurn P, Bawa B, et al: Differential metabolic impact of gastric bypass surgery versus dietary intervention in obese diabetic subjects despite identical weight loss. Sci Transl Med 2011, 3:80re2.

12. Newgard CB, An J, Bain JR, Muehlbauer MJ, Stevens RD, Lien LF, et al A branched-chain amino acid-related metabolic signature that differentiates obese and lean humans and contributes to insulin resistance. Cell Metab 2009, 9:311-326.

13. Tai ES, Tan ML, Stevens RD, Low YL, Muehlbauer MJ, Goh DL, et al: Insulin resistance is associated with a metabolic profile of altered protein metabolism in Chinese and Asian-Indian men. Diabetologia 2010, 53:757-767.

14. Newgard CB: Interplay between lipids and branched-chain amino acids in development of insulin resistance. Cell Metab 2012, 15:606-614.

15. Xu J, Yang S, Cai S, Dong J, Li X, Chen Z: Identification of biochemical changes in lactovegetarian urine using ${ }^{1} \mathrm{H}$ NMR spectroscopy and pattern recognition. Anal Bioanal Chem 2010, 396:1451-1463.

16. May DH, Navarro SL, Ruczinski I, Hogan J, Ogata Y, Schwarz Y, et al: Metabolomic profiling of urine: response to a randomised, controlled feeding study of select fruits and vegetables, and application to an observational study. Br J Nutr 2013, 110:1760-1770.

17. Menni C, Zhai G, Macgregor A, Prehn C, Romisch-Margl W, Suhre K, et al: Targeted metabolomics profiles are strongly correlated with nutritional patterns in women. Metabolomics 2013, 9:506-514.

18. O'Sullivan A, Gibney MJ, Brennan L: Dietary intake patterns are reflected in metabolomic profiles: potential role in dietary assessment studies. Am J Clin Nutr 2011, 93:314-321.

19. Rudkowska I, Paradis AM, Thifault E, Julien P, Tchernof A, Couture P, et al: Transcriptomic and metabolomic signatures of an $n-3$ polyunsaturated fatty acids supplementation in a normolipidemic/normocholesterolemic Caucasian population. J Nutr Biochem 2012, 24:54-61.
20. Bouchard-Mercier A, Paradis AM, Rudkowska I, Lemieux S, Couture P, Vohl MC: Associations between dietary patterns and gene expression profiles of healthy men and women: a cross-sectional study. Nutr J 2013, 12:24.

21. Goulet J, Nadeau G, Lapointe A, Lamarche B, Lemieux S: Validity and reproducibility of an interviewer-administered food frequency questionnaire for healthy French-Canadian men and women. Nutr J 2004, 3:13.

22. Paradis AM, Godin G, Perusse L, Vohl MC: Associations between dietary patterns and obesity phenotypes. Int J Obes (Lond) 2009, 33:1419-1426.

23. Hu FB, Rimm E, Smith-Warner SA, Feskanich D, Stampfer MJ, Ascherio A et al: Reproducibility and validity of dietary patterns assessed with a food-frequency questionnaire. Am J Clin Nutr 1999, 69:243-249.

24. Sherzai A, Heim LT, Boothby C, Sherzai AD: Stroke, food groups, and dietary patterns: a systematic review. Nutr Rev 2012, 70:423-435.

25. Alhazmi A, Stojanovski E, McEvoy M, Garg ML: The association between dietary patterns and type 2 diabetes: a systematic review and meta-analysis of cohort studies. J Hum Nutr Diet 2013. Epub ahead of print

26. Wang TJ, Larson MG, Vasan RS, Cheng S, Rhee EP, McCabe E, et al: Metabolite profiles and the risk of developing diabetes. Nat Med 2011, 17:448-453.

27. Lackey DE, Lynch CJ, Olson KC, Mostaedi R, Ali M, Smith WH, et al: Regulation of adipose branched-chain amino acid catabolism enzyme expression and cross-adipose amino acid flux in human obesity. Am J Physiol Endocrinol Metab 2013, 304:E1175-E1187.

28. Xu HE, Lambert MH, Montana VG, Parks DJ, Blanchard SG, Brown PJ, et al: Molecular recognition of fatty acids by peroxisome proliferator-activated receptors. Mol Cell 1999, 3:397-403.

29. Coll T, Eyre E, Rodriguez-Calvo R, Palomer X, Sanchez RM, Merlos M, et al: Oleate reverses palmitate-induced insulin resistance and inflammation in skeletal muscle cells. J Biol Chem 2008, 283:11107-11116.

30. Stephenson EJ, Camera DM, Jenkins TA, Kosari S, Lee JS, Hawley JA, et al: Skeletal muscle respiratory capacity is enhanced in rats consuming an obesogenic Western diet. Am J Physiol Endocrinol Metab 2012, 302:E1541-E1549.

31. Pranprawit A, Wolber FM, Heyes JA, Molan AL, Kruger MC: Short-term and long-term effects of excessive consumption of saturated fats and/or sucrose on metabolic variables in Sprague Dawley rats: a pilot study. J Sci Food Agric 2013, 93:3191-3197.

doi:10.1186/1475-2891-12-158

Cite this article as: Bouchard-Mercier et al.: The metabolic signature associated with the Western dietary pattern: a cross-sectional study. Nutrition Journal 2013 12:158.

\section{Submit your next manuscript to BioMed Central and take full advantage of:}

- Convenient online submission

- Thorough peer review

- No space constraints or color figure charges

- Immediate publication on acceptance

- Inclusion in PubMed, CAS, Scopus and Google Scholar

- Research which is freely available for redistribution 